\title{
Olfactory cues play a significant role in removing fungus from the body surface of Drosophila melanogaster
}

\author{
$\operatorname{AUTHOR}(\mathrm{S}):$ \\ Yanagawa, Aya; Chabaud, Marie-Ange; Imai, \\ Tomoya; Marion-Poll, Frederic
}

\section{CITATION:}

Yanagawa, Aya ... [et al]. Olfactory cues play a significant role in removing fungus from the body surface of Drosophila melanogaster. Journal of Invertebrate Pathology 2018, 151: 144-150

\section{ISSUE DATE:}

2018-01

URL:

http://hdl.handle.net/2433/237238

\section{RIGHT:}

(C) 2018. This manuscript version is made available under the CC-BY-NC-ND 4.0 license http://creativecommons.org/licenses/by-nc-nd/4.0/:; この論文は出版社版でありません 。引用の際には出版社版をご確認ご利用ください。; This is not the published version. Please cite only the published version. 
1 Olfactory cues play a significant role in removing fungus from the body surface of

2 Drosophila melanogaster

3 Aya Yanagawa ${ }^{1}$, Marie-Ange Chabaud ${ }^{2}$, Tomoya Imai ${ }^{1}$, Frederic Marion Poll ${ }^{3,4}$

4

51 RISH, Kyoto University, Uji city, Japan 611-0011, Japan

62 UMR Physiologie de l'Insecte : Signalisation et Communication, INRA Centre de

7 Versailles, F-78026 Versailles Cedex, France

83 UMR Evolution, Génomes, Comportement, Ecologie, CNRS, IRD, Univ Paris-Sud,

9 Université Paris-Saclay, F-91198 Gif-sur-Yvette, France

104 AgroParisTech, F-75005 Paris, France

11

Abstract

Many insects and Dipterans in particular are known to spend considerable time grooming, but whether these behaviors actually are able to remove pathogenic fungal conidia is less clear. In this study, we examined whether grooming serves to protect flies by reducing the risk of fungal infection in Drosophila melanogaster. First, we confirmed that fungi were removed by grooming. Entomopathogenic, opportunistic, and plant pathogenic fungi were applied on the body surface of the flies. To estimate grooming efficiency, the number of removal conidia through grooming was quantified and we successfully demonstrated that flies remove fungal conidia from their body surfaces via grooming behavior. Second, the roles of gustatory and olfactory signals in fungus removal were examined. The wildtype fly Canton-S, the taste deficiency mutant poxn 70, and the olfactory deficiency mutant orco 1 were used in the tests. Comparisons between Canton-S and poxn 70 flies indicated that gustatory signals do not have a significant role in fungal removal via grooming behavior in $D$. melanogaster. In contrast, the efficiency of conidia removal in orco1 flies was drastically decreased. Consequently, this study indicated that flies rely on mechanical stimulus for the induction of grooming and olfaction for more detailed removal. 


\section{Introduction}

Dipterans spend a significant amount of time grooming (Dawkins and Dawkins, 1976). Grooming behaviors involve brushing the body and the wings with the legs and cleaning the legs and the antenna with the mouthparts. It is reported that grooming may help clean external chemosensory receptors (Böröczky et al., 2013) and contributes to removal of dust particles (Phillis et al., 1993). However, there are very limited data to support the hypothesis that grooming behavior plays a role in the resistance against microbial infection. Most dipterans live in highly humid habitats containing microbes (Rohlfs, 2008) and frequently perform spontaneous grooming (Szebenyi, 1969). It is reported that flies decrease spontaneous exploratory activity when they perceive the presence of other individuals on food resources (Kamyshev et al., 2002). Instead, flies increase individual behaviors, such as preening (when the legs are rubbed together), which are interpreted as signaling movements that maintain flies at a certain minimum distance apart from each other (Connolly, 1968; Kamyshev et al., 2002). Grooming systematically occurs after egg laying (Rieger et al., 2007; Yang et al., 2008). Considering that many microbes can eventually invade insects through their cuticles, self-grooming in Diptera may help to prevent infections from microorganisms living in their habitats.

In insects, hygiene behavior is realized as an integral part of the strategy to cope with pathogens (Vega and Kaya, 2011). If the purpose of grooming is directly linked to the need for cleaning the body from potential ectoparasites, then this behavior may be triggered by signals emanating from microorganisms. Several recent observations performed on social insects indicate that grooming is involved in the resistance against pathogen infection (Zhukovskaya et al., 2013). Spores of entomopathogenic fungi first adhere to the cuticle and then penetrate the surface of the insect by sending hyphae through the epidermis (Yanagawa et al., 2008). Mutual contacts like allogrooming in several species of termites makes them less prone to infection by pathogens (Boucias et al., 1996; Shimizu and Yamaji, 2002; Traniello et al., 2002; Yanagawa and Shimizu, 2007). In honeybees, allogrooming is used to remove debris and parasitic mites (Peng et al., 1987; Bozic and Valentincic, 1995; Rath, 1999). It is also known that ants use grooming to protect themselves from ectoparasites (Tranter and Hughes, 2015; Westhus et al., 2014; Okuno et al., 2012). Drosophila performs self-grooming, although no reports demonstrated the effects of self-grooming on the removal of parasites in Drosophila by using bioassays. Self-grooming is often triggered by touch (Page and Matheson, 2004) or by noxious chemicals (Newland, 1998: Elwood, 2011) detected with nociceptive receptors, which respond to damage or by taste sensilla. The stimulated part of the body or appendage is moved away from the stimulus, and upon increasing stimulation, a 
brushing movement is generated in either of the legs and directed to the site of stimulation (Dürr and Matheson, 2003). Considering these reports, the central nervous system has an important role in generating self-grooming behaviors (Yellman et al., 1997).

We investigated whether self-grooming contributes to preventing infection from fungi in fruit flies, D. melanogaster. First, the susceptibility of the wildtype $D$. melanogaster strain "Canton-S" to three fungal species and isolates: The entompathogen Beuveria bassiana F1286, the opportunist Aspergillus niger ASN5131, and the plant pathogen Fusarium oxisporum $544 \mathrm{H}$ had been tested. Then conidia removal from the Drosophila body surface of all three fungal species of three D. melanogaster strains: The wildtype "Canton-S". The taste mutant strain "poxn $70^{\prime}$. The olfactory deficiency mutant strain "orco1". In this study, we confirmed that flies remove fungal conidia by comparing three strains of fungi with different virulence levels. We then examined the roles of taste and olfactory signals.

\section{Materials and methods}

\subsection{Insects}

Drosophila melanogaster were maintained on a standard cornmeal agar diet and at $20^{\circ} \mathrm{C}$ and $80 \% \mathrm{RH}$. The wildtype strain Canton-S was used for all experiments. The poxn 70 (Yanagawa et al., 2014) and orco1 strains (Bloomington stock \# 23129) were used in the behavioral assays with Beauveria bassiana. In order to establish if these responses were mediated by taste sensilla, we performed the same experiments on flies deprived of their external taste chemoreceptors by means of a poxn 70 mutation, which deters development of external chemoreceptors (Nottebohm et al., 1994). To investigate the importance of olfactory perception on fungal removal, we used orco1 mutant flies. Or $83 b$ is abolished in orco1 mutant flies. This protein is essential for Drosophila olfaction (Lausson et al., 2004). Four-day-old flies were used in all experiments. All experiments were conducted in a room without window and under normal room light. All rooms were maintained at $23-26{ }^{\circ} \mathrm{C}$. Flies were placed in the experiment room for about one hour before use to get use to the new environment so that the light in the test room was not affecting the behavior of the insect.

\subsection{Fungi and preparation of conidial suspension}

Three different fungi were used in our experiments: Beauveria bassiana, Aspergillus niger, and Fusarium oxysporum. B. bassiana is an entomopathogenic fungus, which is 
109 known to infect Drosophila (Clarkson and Charnley, 1996; Lemaitre et al., 1997). A. niger 110 is an opportunistic microbe (Klainer and Beisel, 1969) and F. oxysporum is a plant 111 pathogen (Snyder and Hansen, 1940).

112 Laboratory maintained isolates were used for the experiments. B. bassiana F1286 was 113 maintained on L-broth agar (1\% polypeptone, $0.3 \%$ yeast extract, $2.0 \%$ sucrose, $0.5 \%$ $114 \mathrm{NaCl}$, and $2.0 \%$ agar) at $25^{\circ} \mathrm{C}$. A. niger ASN5131 and $F$. oxysporum $544 \mathrm{H}$ were 115 maintained on potato dextrose agar (PDA) (0.4\% potato extract, $2.0 \%$ glucose, and $1.5 \%$ 116 agar) at $25^{\circ} \mathrm{C}$. Conidia were harvested from 10-day-old to 15 -day-old cultures using a 117 brush and were suspended in various solutions as follows. The conidial suspensions (A 118 series) of all fungal strains were prepared in a $0.025 \%$ aqueous solution of Tween 20 to 119 evaluate virulence. These solutions were diluted $10^{1}, 10^{2}, 10^{3}$, and $10^{4}$ times. On each 120 PDA petri dish, $0.1 \mathrm{ml}$ of the diluted suspension was pipetted and then spread using a sterilized glass spreader. The Petri dishes were incubated at $25^{\circ} \mathrm{C}$ for 3 days. The numbers of colony-forming units per milliliter (CFU/ml) were determined on the basis of the numbers of colonies on these PDA plates. To detect the conidia on the cuticles, the conidia were surface-labeled with $0.01 \%$ fluorescein isothiocyanate solution (FITC, Sigma Chemical) according to the protocols outlined by Hung and Boucias (1992). The FITC-labeled conidia in a $0.025 \%$ aqueous solution of Tween 20 were counted using a Thoma hemocytometer (Erma Inc, Japan) and adjusted to a concentration of $1.0 \times 10^{7}$ conidia/ml (B series). Over $95 \%$ of viability was confirmed on both series of conidia suspension.

\subsection{Fly susceptibility to fungal infection}

132 We first tested the susceptibility of the flies to each fungal strain. For inoculation, 133 Canton-S flies were collected and placed on ice for 3-5 minutes to induce light anesthesia. 134 The flies were then placed in microcentrifuge tubes containing the conidial suspensions 135 (A series) (A. niger, $1.63 \times 10^{7} \mathrm{CFUs} / \mathrm{ml} ; F$ oxysporum, $1.30 \times 10^{7} \mathrm{CFUs} / \mathrm{ml}$; and $B$. 136 bassiana, $6.25 \times 10^{8} \mathrm{CFUs} / \mathrm{ml}$ ). The flies were submerged in conidial suspensions with 137 gentle swirling for 5 seconds and allowed to dry on a Whatman No. 1 filter paper. When 138 they recovered from anesthesia and started to move, a group of 10 flies (5 male and 5 139 female) were transferred on a filter paper disc to $90 \times 15 \mathrm{~mm}$ Petri dishes and fly medium

140 in a cup $(10 \times 5 \mathrm{~mm})$. Flies treated only with a $0.025 \%$ aqueous solution of Tween 20 were 141 reared as controls. They were incubated at $25^{\circ} \mathrm{C}$ and $60 \% \mathrm{RH}$ in the dark room. Mortality 142 and median lethal dose $\left(\mathrm{LD}_{50}\right)$ values were calculated seven days after inoculation. 
Flies were inoculated with the FITC-labeled conidial suspensions (B series), as described above. After treatment with FITC-labeled conidia, the flies were incubated at $25^{\circ} \mathrm{C}$. At intervals of $0,3,24,48$, and 72 hours, 10 flies were removed and stored at $20^{\circ} \mathrm{C}$. Flies were carefully mounted in a drop of Vectashield (Vector Laboratories, USA) to stabilize the fluorescence and were examined using an epifluoresence microscope (Axioplan, Carl Zeiss, Germany) at 200×magnification through a common UV filtering cubes FT510. Photos were taken with a charge-coupled device camera (DP74, Olympus, Japan). Four defined sites (head, thorax, wing, and abdomen) were examined on each fly for attachment of conidia, which was calculated in relation to the whole body. To compare the attachment and persistence of the three different fungi (A. niger, F. oxysporum, and $B$. bassiana), the number of conidia on the insect body surface was counted. We then examined the abilities of the three different Drosophila strains (wild-type fly Canton-S, taste deficient mutant poxn70, and olfactory deficient mutant orco1) to remove conidia. The B. bassiana suspension (B series) was used to compare fungus removal ability in flies, as it had the best initial attachment. Removal efficiency for the initial attachment was compared using the removal index (RI) (number of conidia attached to the insect body surface at each time interval)/(number of conidia initially attached to the insect body surface).

163

\subsection{Taste signals and the induction of grooming}

Grooming induction was assayed in decapitated four-day-old Canton-S flies using the method described by Yanagawa et al. (2014). Olfaction is perceived by antennae and maxillary palps, and gustation is perceived by the proboscis, legs, wings, and genitalia (Vosshall and Stocker, 2007). Since decapitated flies were employed in this test, the influence of olfaction was ruled out and only taste signals were examined. Decapitated flies are capable of self-grooming movements either spontaneously or following specific stimulation, such as touching. These movements mostly involve the meta-thoracic legs, which are raised and moved independently in a succession of strokes. The legs brush the wings, abdomen, and dorsum, or are extended under the abdomen and touch each other in a series of reciprocal sliding movements. Flies were placed on ice for $3-5$ minutes to induce light anesthesia. They were then placed under a stereoscope. Ten flies were then decapitated using a single cut at the neck made by micro-scissors. The decapitated flies then awoke over the next 2-3 minutes. They were placed in an upright position and allowed to recover. In order to stimulate the flies, the wings, forelegs, or hindlegs were gently touched using a sharpened toothpick previously soaked in a test solution. The test 
181

182

183

184

185

186

187

188

189

190

191

192

193

194

195

196

197

198

199

200

201

202

203

204

205

206

207

208

209

210

211

212

213

214

215

216

and B. bassiana F1286 (A series). They were counted using a Thoma hemocytometer and adjusted to a concentration of $1.0 \times 10^{7}$ conidia/ml. These solutions were diluted $10^{1}, 10^{2}$, $10^{3}$, and $10^{4}$ folds to examine the concentration-dependence of the reaction. The bioassays were performed at room temperature on standing flies placed on a piece of paper. The room temperature kept at about $20^{\circ} \mathrm{C}$. Grooming behavior after touching by the toothpick was observed and quantified by a scale. A score of 0 indicates no behavioral induction, a score of 1 indicates 1-2 grooming behaviors (or less than 10 seconds), a score of 2 indicates 3-6 grooming behaviors (or less than 20 seconds), and a score of 3 indicates a strong grooming induction (more than 20 seconds). Twenty female and 20 male flies were tested for each fungus.

\subsection{Olfactory signals and fungal removal}

First, grooming performance was tested in orco1 flies, since they failed to remove fungal conidia from their body surface. To confirm this, we treated flies with chalk powder and examined whether they could clean the dust. Visual comparisons were made with Canton-S flies 6 hours after treatment.

We then determined whether fungal odor enhances or induces the hygiene behavior in both Canton-S flies and orco1 flies. Since B. bassiana is the representative entomopathogenic fungus to D. melanogaster and its conidia attachment and removal efficiency are the largest in three tested fungi as well, we have used B. bassiana for this test. The GC profile of the fungal odor is also available only on B. bassiana from previous study (Yanagawa et al., 2011). Three to five intact Drosophila were placed in a 20-ml vial and exposed to control air for at least 10 minutes. Stimulus air containing B. bassiana odor was then provided for 3 minutes. Spontaneous grooming was observed for 3 minutes prior to the onset of the stimulus. The time intervals that a sample fly devoted to grooming behavior were added to obtain a numeral conversion for grooming.

Airflow was controlled using a three-way cock. Two sides of the cock were connected to a bottle $(30 \mathrm{ml})$ that contained an odor source. One side of the cock was connected to air from $1 \mathrm{ml}$ of $1.0 \times 10^{7} / \mathrm{ml} \mathrm{B}$. bassiana conidial suspension, and the other side was connected to $1 \mathrm{ml}$ of $0.025 \%$ Tween 20 solution as a control. Stimulus and control air both flowed into the three-way cock and the air offered to the flies was regulated by the cock. Fresh air was pumped into the system using a diaphragm pump (AP-115 Iwaki air pump; Iwaki Co., Ltd., Japan) and cleaned through serially connected bottles containing silica gel, molecular sieves $3 \mathrm{~A}$ and 5A, and active carbon. The cleaned airflow was divided into two channels using a Y-shaped connector. Each air channel was connected to a bottle (30 
$217 \mathrm{ml}$ ) that contained one of the odors being tested. This bottle was then connected to one

218 side of the three-way cock. The flow in each channel was regulated to $400 \mathrm{ml} / \mathrm{minute}$ 219 using an inline flowmeter. Twenty flies were examined per experiment. These 220 experiments were carried out in the laboratory, and test arena was maintained at $20^{\circ} \mathrm{C}$ and about $69 \% \mathrm{RH}$ under room light conditions.

222

\subsection{Fungal avoidance}

Since avoidance is another major hygiene behavior aimed at preventing infection, we determined whether chemical signals induce any reactions in different behavioral paradigms involving the same fungi. To assess avoidance due to chemical signals, the visitation test, as described by Marella et al. (2006), was used, with modifications (Supplementary Fig. S1). Canton-S flies were starved for 22 hours using a wet filter paper disc and were transferred to cylindrical containers (height, $7 \mathrm{~cm}$; diameter, $3 \mathrm{~cm}$, polystyrene). The tube bottom was separated into two parts and each part was filled with $1 \mathrm{ml}$ of $1 \%$ agarose containing $100 \mathrm{mM}$ sucrose. The surface of one side was treated with $20 \mathrm{pl}$ of the tested solutions $\left(1.0 \times 10^{7}\right.$ conidia/ml of $A$. niger, $F$. oxysporum, and $B$. bassiana), and the other side was treated with $20 \mu \mathrm{l}$ of a $0.025 \%$ aqueous solution of Tween 20. The solutions were spread onto the filter paper using a spreader. In the control set, both sides were treated with a $0.025 \%$ aqueous solution of Tween 20 . For the negative control set, one side was treated with $20 \mathrm{\mu l}$ of $10^{-1} \mathrm{M}$ quinine, and the other side was treated with $20 \mu \mathrm{l}$ of $0.025 \%$ aqueous solution of Tween 20 . Approximately 40 flies were placed in a bottle and allowed to explore the agarose for 30 minutes. The visitation rate was estimated by providing flies access to agar on the bottom of a test tube. One-half of the agar was treated with a chemical and the other was not. By sampling the number of flies in each area at regular intervals (every 30 seconds over 30 minutes), we can compute a mean preference index $(\mathrm{PI}=(\mathrm{n} 1-\mathrm{n} 2) /(\mathrm{n} 1+\mathrm{n} 2))$ and monitor the number of flies visiting both substrates $(n 1+n 2)$. The number of flies on each side was recorded every 30 seconds using digital photographs, which were then manually counted. Data were obtained from 10 replicates for each substance.

\subsection{Statistical analysis}

248 We used multiple logistic regression analysis to examine conidia removal from the insect surface and concentration-dependent increases in grooming behavior in

250 decapitated flies with respect to sex, chemicals, and fly strains. Dunnett's tests were used

251 to compare RI values used to determine conidia removal efficiency from the initial 252 attachment, and PI values used to compare preferences in in the visiting test. To 
determine the odor-induced increase in grooming, Kruskal-Wallis tests were used to compare the time that flies dedicate to each behavior. JMP 10.0 software (SAS) was used for all analyses.

256

257

\section{Results}

258

3.1 Fly susceptibility

Mortality at one week rearing was as follows. B. bassiana: $67 \%$, A niger: $25 \%, F$. oxysporum: $0 \%$, and controls: $0 \%$. The $\mathrm{LD}_{50}$ values of the fungi in D. melanogaster were B. bassiana F1286: $\geq 4.16 \times 10^{6} \mathrm{CFU} / \mathrm{ml}$, A. niger ASN5131: $>1.63 \times 10^{7} \mathrm{CFU} / \mathrm{ml}$, and $F$. oxysporum $544 \mathrm{H}:>1.295 \times 10^{7} \mathrm{CFU} / \mathrm{ml}$. Drosophila were more susceptible to B. bassiana than $A$. niger and F. oxysporum. The LD50 values are provided in Supplementary Table S1.

3.2 Attachment and removal of fungal conidia on the Drosophila cuticle

The binding of the FITC-labeled conidia to the defined sites on the surfaces of the flies was quantified using an epifluoresence microscope. Attachment and persistence of FITClabeled conidia on the fly cuticle are illustrated in Fig. 1 according to fungal strain, time, and sites of attachment. There was a significant reduction in the number of attached conidia on the insect surface ( $B$. bassiana on Canton-S flies: $\mathrm{p}<0.01, \mathrm{~F}=60.32 ; A$. niger on Canton-S flies: $\mathrm{p}<0.01, \mathrm{~F}=18.10$; and F. oxysporum on Canton-S flies: $\mathrm{p}<0.01, \mathrm{~F}=$ 44.22 ; logistic regression). There was no sex difference in conidium removal efficiency ( $p$ $>0.1$ in all strains on the entire body surface). The number of attached conidia at the initial stage clearly reflected fungal virulence. $B$. bassiana conidia has higher attachment than the other strains (Figs. 1 and 2). Both Canton-S flies and poxn 70 flies removed the conidia to a similar extent ( $B$. bassiana on poxn flies: $\mathrm{p}<0.01, \mathrm{~F}=61.74$ ) (Fig. 3). In contrast, orco1 flies failed to remove the conidia (B. bassiana on orco1 flies: $\mathrm{p}$ $>0.01, F=61.74$ ) (Fig. 3). Sex differences were observed only in Canton-S flies at the wing site (Supplementary Fig. S2). More conidia stayed on the wings in female flies. This indicates that female flies rely more on both gustatory and olfactory signals to remove fungi from the wings when compared to the male flies (Student $\mathrm{T}$ test: $\mathrm{p}<0.05$ at all time intervals) (Supplementary Fig. S2). This difference was not observed in poxn 70 or orco1 flies (Student T test: $\mathrm{p}>0.1$ at all time intervals).

We scored grooming responses following contact with the tip of a small wood stick 
289

290

291

292

293

294

295

296

297

298

299

300

301

302

303

304

305

306

307

308

309

310

311

312

313

314

315

316

317

318

319

320

321

322

323

324

into contact with the margins of the wings, the front legs, or the hind legs. We first tested the different fungal suspensions (F1286, ASN5131, and 544H). None of the fungal suspensions induced grooming in the flies (B. bassiana F1286: foreleg, concentration, $\mathrm{X}^{2}$ $=0.399, \mathrm{p}=0.983 ;$ sex, $\mathrm{X}^{2}=0.540, \mathrm{p}=0.970$; hind leg, concentration, $\mathrm{X}^{2}=4.658, \mathrm{p}=$ 0.324 ; sex, $X^{2}=7.040, p=0.134$; wing, concentration, $X^{2}=7.886, p=0.096$; sex, $X^{2}=3.529$, $\mathrm{p}=0.474 ;$ A niger ASN5131: foreleg, concentration, $\mathrm{X}^{2}=1.936, \mathrm{p}=0.748 ;$ sex, $\mathrm{X}^{2}=0.235$, $\mathrm{p}=0.994$; hind leg, concentration, $\mathrm{X}^{2}=1.819, \mathrm{p}=0.769 ;$ sex, $\mathrm{X}^{2}=2.109, \mathrm{p}=0.716$; wing, concentration, $\mathrm{X}^{2}=0.627, \mathrm{p}=0.959 ;$ sex, $\mathrm{X}^{2}=2.144, \mathrm{p}=0.709 ;$ F. oxysporum $544 \mathrm{H}$ : foreleg, concentration, $\mathrm{X}^{2}=6.566, \mathrm{p}=0.161$; sex, $\mathrm{X}^{2}=7.687, \mathrm{p}=0.104$; hind leg, concentration, $X^{2}=2.335, p=0.674 ;$ sex, $X^{2}=2.464, p=0.651$; wing, concentration, $X^{2}=4.045, p=0.400 ;$ sex $X^{2}=6.876, p=0.143$; logistic regression).

\subsection{Olfactory signals in fungal removal}

Flies successfully cleaned the chalk dust from their bodies. There was no visible difference in the cleaning of the dust between Canton-S flies and orco1 flies. This indicates that olfaction does not influence dust removal.

Grooming induced by fungal odor was examined using the odor exposure test. Behaviors of Drosophila during the air exposure experiments are illustrated in Fig. 4. In addition to grooming, two new conditions were observed; 1) 'stay' which means no moving (standing still) and 2) 'activity', which encompasses all other movements except from grooming. Mostly, flies walked or ran in 'activity' status. Since there was significant difference in grooming behavior between females and males (grooming, $\mathrm{X}^{2}=10.641, \mathrm{p}=$ 0.001; stay, $\mathrm{X}^{2}=5.367, \mathrm{p}=0.023$; activity, $\mathrm{X}^{2}=5.367, \mathrm{p}=0.023$; Kruskal-Wallis test), behaviors were analyzed by females and males independently. We observed more running behavior in female Canton-S flies ( $x^{2}=8.526, p=0.004$, Kruskal-Wallis test), however, no other significant behavior effect was observed during exposure to the harmful fungus air (Canton-S flies_female: grooming, $X^{2}=0.047, p=0.829$; stay, $X^{2}=$ $0.6812, \mathrm{p}=0.409$; activity, $\mathrm{X}^{2}=1.294, \mathrm{p}=0.255$; Canton-S flies_male: grooming, $\mathrm{X}^{2}=$ $0.019, \mathrm{p}=0.892 ;$ stay, $\mathrm{x}^{2}=1.657, \mathrm{p}=0.198 ;$ activity, $\mathrm{X}^{2}=8.526, \mathrm{p}=0.004 ;$ orco 1 flies_female: grooming, $\mathrm{X}^{2}=0.001, \mathrm{p}=0.978$; stay, $\mathrm{X}^{2}=0.106, \mathrm{p}=0.745$; activity, $\mathrm{X}^{2}=$ $1.058, \mathrm{p}=0.304$; orco1 flies_male: grooming, $\mathrm{X}^{2}=0.105, \mathrm{p}=0.745 ;$ stay, $\mathrm{X}^{2}=0.009, \mathrm{p}=$ 0.925 ; activity, $\mathrm{X}^{2}=0.000, \mathrm{p}=1.000 ;$ Kruskal-Wallis test).

\subsection{Fungal avoidance}

No sex differences were found in the PI indexes ( $p=0.45$, analysis of variance, Fig. 5). The PI measured during the control treatment was $0.04 \pm 0.04$. The flies visited both 
sides of the non-treated agar equally and exhibited a strong aversion to quinine in the negative control test $(\mathrm{PI}=-0.71 \pm 0.06, \mathrm{p}<0.001$, Dunnett's test). The flies did not typical preference or avoidance behaviors in response to any of the fungal suspensions $(B$. bassiana: $\mathrm{PI}=0.10 \pm 0.07, \mathrm{p}=0.14 ;$ A niger: $\mathrm{PI}=-0.11 \pm 0.04, \mathrm{p}=0.92 ;$ and F. oxysporum: $\mathrm{PI}=-0.06 \pm 0.04, \mathrm{p}=1$ in Canton-S flies; $B$. bassiana: $\mathrm{PI}=0.01 \pm 0.06, \mathrm{p}=1$ in poxn flies; Dunnett's test).

\section{Discussion}

Grooming behavior seems to have diverse roles. Indeed, many factors involved in this behavior are still unknown. In this study, we examined the roles of gustatory and olfactory signals on fungus removal. First, we successfully demonstrated that flies remove fungal conidia from their body surfaces via grooming behavior. Comparisons between wildtype Canton-S flies and the chemical mutants poxn 70 and orcol indicated that gustatory signals do not have a significant role in fungal removal via grooming behavior in D. melanogaster, although olfactory signals are involved in this behavior. It seems that flies rely on mechanical stimulation for the induction of grooming and on olfaction for more detailed removal.

D. melanogaster remove microbes, such as ectoparasites, from their surfaces via grooming behavior (Fig. 1). The flies removed conidia from all fungal strains.

Differences in the initial attachment numbers for each strain, which reflect the virulence levels of the different fungi, support our previous findings that attachment ability is important in estimating fungal virulence (Yanagawa et al., 2008). FITClabelled fungal conidia enabled us to visualize fungal ectoparasites and monitor their behavior on the host surface. The design of the bioassay was another key for the quantitative observation of conidial removal. Spraying has usually been used to apply fungi onto flying insects. However, this method requires large amounts of conidial solution, which are difficult to produce at the laboratory level (Ingris et al., 2012). Moreover, the Drosophila rearing conditions used (vial with a medium-covered bottom) (Greenspan, 2004) prevented us from using other methods, such as immersion or droplet application, which are usually used for beetles. These methods created humidity levels that are too high for flies to survive. Indirect applications, such as embrocation using a soft brush, which is usually used for worms, are also problematic, as they may lead to damage to the wings of the flies. We avoided all of the above problems by using a flat arena (Supplementary Fig. S3). After the flies were immersed in the conidial suspension, they were able to dry themselves on the filter paper and came into contact with wet food after they were fully dried. 
Grooming seems to be triggered by mechanoreceptors (Page and Matheson, 2004) or taste sensilla (Newland, 1998) in most other insects. However, many recent studies have reported that odors from bacteria and yeast modulate fly behavior. These odors are detected by $D$. melanogaster using specialized olfactory receptor proteins (Becher et al., 2012; Stensmyr et al., 2012; Kapsetaki et al., 2014; Dweck et al., 2015; Falchi et al., 2015). Comparisons of conidia removal in Canton-S flies and orco1 flies indicate that olfactory signals play a significant role in the removal of $B$. bassiana conidia from the Drosophila body surface. The fact that orco1 mutants were able to clear up chalk powder indicates that there may be a unique role for olfactory cues in fungus removal. Experiments using poxn flies indicate that taste signals are not important in removing fungal conidia from the body surfaces of $D$. melanogaster, as poxn 70 flies display almost the same conidia removal efficiency as Canton-S flies. Moreover, there was no grooming induction by fungus-related taste stimuli. We have demonstrated that gustatory stimuli from bacteria are involved in grooming reflexes (Yanagawa et al., 2014). The results of the grooming induction test in this study therefore indicate that Drosophila use microbial signals from $E$. coli and fungi differently in the induction of grooming behavior. This is because gustatory signals from suspensions of $E$. coli induce grooming while the same is not true of suspensions of fungi. Phillis et al. (1993) have reported detailed grooming induced by mechanical stimuli in $D$. melanogaster. Conidia were attached everywhere on the surface of the flies, and some $B$. bassiana conidia were attached directly to sensory hairs. This observation supports the role of mechanoreceptors in fungal grooming. In addition, considering the success of the orco flies in removing chalk powder, it seems that removal of foreign objects via grooming mainly relies on mechanical stimulation. Conidial attachment most likely leads to mechanical stimulation, which then induces the removal of all foreign organisms on the insect's surface. In Canton-S flies, however, the more highly virulent strain, $B$. bassiana, was more carefully removed, as the conidia reduction was significant at alltime intervals. The higher level of initial attachment was persistent (Fig. 1). Although the numbers of conidia decrease substantially over time, a marked reduction was observed in the numbers of FITC-labeled conidia associated with virulence. Notably, significant differences were observed in conidium removal from the wings between the two sexes in Canton-S flies, but not in poxn70 or orco1 flies. This supports the idea that both taste and olfactory signals are used for fungal cleaning in intact flies, especially in female flies.

Flies usually do not move in the direction of harmful microbial odors (Stensmyr et al. 2012). Although we do not yet know whether flies possess specialized olfactory receptor 
397

398

399

400

401

402

403

404

405

406

407

408

409

410

411

412

413

414

415

416

417

418

419

420

421

422

423

424

425

426

427

428

429

proteins to the volatile compounds of $B$. bassiana, in our previous study, we detected 1octen-3ol in odors from B. bassiana (Yanagawa et al., 2011). This compound is a wellknown aversive odorant to flies (Silbering et al., 2011). This may explain the higher levels of running/walking activity in female Canton-S flies after exposure to the musty odor. It is reported that termites generally avoid odors from entomopathogenic fungi, but move toward these odors to remove it when they sense the presence of pathogens nearby (Yanagawa et al., 2015). The odor from the pathogenic mite fungus Neozygites floridana is known to be an attractive signal for males upon their mating and facilitates the transmission of the fungus to healthy individuals (Trandem et al., 2015). This suggests that fungal signals have differing significance to host insects when they are mixed with other odors based on the insect's condition/situation. It is possible that fungi have also developed the ability of using insect perception during their evolution and produce or potentially modify their odors. Fungal odors are known to attract Drosophila larvae when the fungal colony is still young (Rohlfs, 2005). Since they have more interactions with general contaminating fungi, the insects may rely on fungal odors to find food. Nevertheless, the manner by which insects perceive microbes is still ambiguous. Insect behavioral reactions to microbial signals may be regulated by the delicate balance between neural regulatory pathways that perceive odors as beneficial signals denoting a food source, oviposition site, or mating individual, and those perceiving odors as harmful signals denoting microbial infection.

Insects often groom themselves spontaneously. This grooming behavior is increased following the introduction of environmental changes, such as those caused by changes in odor, taste, air, light, or physical contact (Zhukovskaya et al., 2013). The factors involved in this behavior are varied. It was interesting that $D$. melanogaster were found to possess different neural cascades used to trigger grooming by different types of microbe. More research on how insects use signals from microbes will lead to a broader understanding of ecological interactions in nature.

\section{Acknowledgement}

This work was supported by the Future Development Funding Program of Kyoto University Research Coordination Alliance and the mission research program of 
430 Research Institute for Sustainable Humanosphere in Kyoto University(grant

431 number:2016-5-1-5).

432

433

References

434

Awasaki T, Kimura K-I (1997) pox-neuro is required for development of chemosensory

435

436

437

438

439

440

441

442 bristles in Drosophila. J Neurobiol 32, 707-721.

Becher PG, Flick G, Rozpędowska E, Schmidt A, Hagman A, Lebreton S, Larsson MC, Hansson BS, Piškur J, Witzgall P, Bengtsson M (2012) Yeast, not fruit volatiles mediate Drosophila melanogaster attraction, oviposition and development, Functional Ecology 26, 822-828.

Böröczky K, Wada-Katsumata A, Batchelor D, Zhukovskaya M, Schal C (2013) Insects groom their antennae to enhance olfactory acuity. Proceedings of the National Academy of Sciences of the United States of America 110, 3615-3620.

Boucias DG, Stokes C, Storey G, Pendland JC (1996) The effects of imidacloprid on the termites Reticulitermes flavipes and its interaction with the mycopathogen Beauveria bassiana. Pflanzenschutz-Nachr. Bayer 49, 103-144.

Bozic J, Valentincic T (1995) Quantitative analysis of social grooming behavior of the honey bee Apis mellifera carnica. Apidologie, 26, 141-147.

Clarkson JM, Charnley AK (1996) New insights into the mechanisms of fungal pathogenesis in insects, Trends Microbiol. 4(5), 197-203.

Connolly,K (1968) Thesocialfacilitationofpreeningbehaviourin Drosophila melanogaster. Anim.Behav. 16, 385-391.doi:10.1016/0003-3472(68)90023-7

Dawkins R, Dawkins M (1976) Hierarchical organization and postral facilitation: rules for grooming in flies, Anita. Behav. 24, 739-755.

Dürr V, Matheson T. (2003) Graded limb targeting in an insect is caused by the shift of a single movement pattern. Journal of Neurophysiology 90, 1754-1765.

Dweck HK, Ebrahim SA, Farhan A, Hansson BS, Stensmyr MC (2015) Olfactory proxy detection of dietary antioxidants in Drosophila, Curr Biol. 25, 455-466.

Elwood RW (2011) Pain and Suffering in Invertebrates? ILAR Journal 52, 175-184.

Falchi G, Marche MG, Mura ME, Ruiu L (2015) Hydrophobins from aerial conidia of Beauveria bassiana interfere with Ceratitis capitata oviposition behavior, Biological Control 81, 37-43.

French A, Agha MA, Mitra A, Yanagawa A, Sellier M-J, Marion-Poll F (2015) Drosophila bitter taste(s), Front. Integr. Neurosci. 9, article 58.

Greenspan RJ (2004) Fly Pushing: The Theory and Practice of Drosophila Genetics 2nd 465 Edition, Cold Spring Harbor Laboratory Press, New York, pp191. 
466

467

468

469

470

471

472

473

474

475

476

477

478

479

480

481

482

483

484

485

486

487

488

489

490

491

492

493

494

495

496

497

498

499

500

501

Hung ST, Boucias DG (1992) Influence of Beauveria bassiana on the cellular defense response of the beet armyworm, Spodoptera exigua. J. Invertebr. Pathol. 60, 152158.

Inglis GD, Enkerli J., Goettel MS (2012) Chapter VII Laboratory techniques used for entomopathogenic fungi: hypocreales, In Manual of techniques in invertebrate pathology second edition, edited by Lacey LA, Washington, USA, Academic Press, 189-253.

Kamyshev NG, Iliadi KG, Bragina JV, Kamysheva EA, Tokmatcheva EV, Preat T, Savvateeva-Popova EV (2002) Novel memory mutants in Drosophila: Behavioral characteristics of the mutant nemy ${ }^{\mathrm{P} 153}$, BMC Neuroscience 3: 9, http://www.biomedcentral.com/1471-2202/3/9

Kapsetaki S-E, Tzelepis I, Avgousti K, Livadaras I, Garantonakis N, Varikou K, Apidianakis Y (2014) The bacterial metabolite 2-aminoacetophenoneromotes association of pathogenic bacteria with flies, Nature Communications 5, article number: 4401.

Klainer AS, Beisel WR (1969) Opportunistic infection: A review, American Journal of the Medical Sciences 258, 431-456.

Lausson MC. Domingos AI, Jones WD, Chiappe ME, Amrein H, Vosshall LB (2004) Or83b encodes a broadly expressed odorant receptor essential for Drosophila olfaction, Neuron 43, 703-714.

Lemaitre B, Reichhart J-M, Hoffmann JA (1997) Drosophila host defense: Differential induction of antimicrobial peptide genes after infection by various classes of microorganisms, Proc. Natl. Acad. Sci. 94, 14614-14619.

Marella S, Fischler W, Kong P, Asgarian S, Rueckert E, Scott K. (2006) Imaging taste responses in the fly brain reveals a functional map of taste category and behavior. Neuron 49, 285-295.

Newland PL (1998). Avoidance reflexes mediated by contact chemoreceptors on the legs of locusts. J Comp Physiol A 183, 313-324.

Nottebohm E, Usui A, Therianos S, Kimura K, Dambly-Chaudière C, Ghysen A. (1994) The gene poxn controls different steps of the formation of chemosensory organs in Drosophila. Neuron 12, 25-34.

Okuno M, Tsuji K, Sato H, Fujisaki K (2012) Plasticity of grooming behavior against entomopathogenic fungus Metarhizium anisopliae in the ant Lasius japonicus, Journal of Ethology 30, 23-27.

Page KL, Matheson T (2004) Wing hair sensilla underlying aimed hindleg scratching of the locust. J Exp Biol 207, 2691-2703. 
502

503

504

505

506

507

508

509

510

511

512

513

514

515

516

517

518

519

520

521

522

523

524

525

526

527

528

529

530

531

532

533

534

535

536

537

Peng YS, Fang Y, Xu S, Ge L, Nasr ME (1987) The resistance mechanism of the Asian honey bee, Apis cerana Fabr, to an ectoparasitic mite Varroa jacobsoni Oudemans, J. Invertebr. Pathol. 49, 54-60.

Phillis RW, Bramlage AT, Wotus C, Whittaker A, Gramates LS, Seppala D, Farahanchi F, Caruccio P, Murphey RK (1993). Isolation of mutations affecting neural circuitry required for grooming behavior in Drosophila melanogaster. Genetics 133, 581-592.

Rath, W. (1999) Co-adaptation of Apis cerana Fabr. and Varroa jacobsoni Oud. Apidologie 30, 97-110.

Rieger D, Fraunholz C, Popp J, Bichler D, Dittmann R, Helfrich-Forster C (2007) The fruit fly Drosophila melanogaster favors dim light and times its activity peaks to early dawn and late dusk. J Biol Rhythms 22, 387-399.

Rohlfs M (2005) Clash of kingdoms or why Drosophila larvae positively respond to fungal competitors, Frontiers in Zoology 2, article 2.

Rohlfs M (2008) Host-parasitoid interaction as affected by interkingdom competition, Oecologia 155, 161-8.

Shimizu S, Yamaji M (2002) Pathogenicity of entomopathogenic fungi to the termite, Reticulitermes speratus. Jpn. J. Appl. Entomol. Zool. 46, 89-91 (in Japanese with English summary).

Snyder WC, Hansen HN (1940) The species concept in Fusarium, Botany American Journal of Botany 27 (2), 64-67.

Stensmyr MC, Dweck HKM, Farhan A, Ibba I, Strutz A, Mukunda L, Linz J, Grabe V, Steck K, Lavista-Llanos S, Wicher D Sachse S, Knaden M, Becher PG, Seki Y, Hansson BS (2012) A conserved dedicated olfactory circuit for detecting harmful microbes in Drosophila, Cell 151, 1345-1357.

Szebenyi AL (1969) Cleaning behaviour in Drosophila melanogaster, Animal Behaviour $17,641-651$.

Trandem N, Bhattarai UR, Westrum K, Knudsen GK, Klingen I (2015) Fatal attraction: Male spider mites prefer females killed by the mite-pathogenic fungus Neozygites floridana, Journal of Invertebrate Pathology 128, 6-13.

Traniello JFA, Rosengaus RB, Savoie K (2002) The development of immunity in a social insect: Evidence for the group facilitation of disease resistance. Proceedings of the national academy of science of the United States of America 99, 6838-6842.

Tranter C, Hughes WOH (2015) Acid, silk and grooming: alternative strategies in social immunity in ants?, Behavioral Ecology and Sociobiology 69, 1687-1699.

Vega F, Kaya H (2011) Insect Pathology second edition, Academic Press, San Diego, pp 
538

539

540

541

542

543

544

545

546

547

548

549

550

551

552

553

554

555

556

557

558

559

560

561

562

563

564

565

566

567

568

569

570

571

572

573

508.

Vosshall LB, Stocker RF (2007) Molecular Architecture of Smell and Taste in Drosophila, Annual Review of Neuroscience 30, 505-533.

Westhus C, Ugelvig LV, Tourdot E, Heinze J, Doums C, Cremer S (2014) Increased grooming after repeated brood care provides sanitary benefits in a clonal ant, Behavioral Ecology and Sociobiology 68, 1701-1710.

Yanagawa A, Shimizu S (2007) Resistance of the termite, Coptotermes formosanus Shiraki to Metarhizium anisopliae due to grooming. BioControl 52 (1), 75-85.

Yanagawa A, Yokohari F, Shimizu S (2008) Defense mechanism of the termite, Coptotermes formosanus Shiraki, to entomopathogenic fungi, J Invertebr Pathol 97, 165-170.

Yanagawa A, Fujiwara-Tsujii N, Akino T, Yoshimura T, Yanagawa T, Shimizu S (2011) Musty odor of entomopathogens enhances disease-prevention behaviors in the termite Coptotermes formosanus, Journal of Invertebrate Pathology 108, 1-6.

Yanagawa A, Guigue A, Marion-Poll F (2014) Hygienic grooming is induced by contact chemicals in Drosophila melanogaster, Front. Behav. Neurosci. 8, article 254.

Yanagawa A, Imai T, Akino T, Toh Y, Yoshimura T (2015) Olfactory cues from pathogenic fungus affect the choice of moving direction of termites, Coptotermes formosanus, Journal of Chemical Ecology 41, 1118-1126.

Yang C-h, Belawat P, Hafen E, Jan LY, Jan Y-N. (2008) Drosophila egg-laying site selection as a system to study simple decision-making processes. Science 319, 1679-1683.

Yellman C, Tao H, He B, Hirsh J (1997) Conserved and sexually dimorphic behavioral responses to biogenic amines in decapitated Drosophila. PNAS 94, 4131-4136.

Zhukovskaya M, Yanagawa A, Forschler BT (2013) Grooming Behavior as a Mechanism of Insect Disease Defense, Insects 4, 609-630.

\section{Figure legends}

Fig. 1. Attachment and persistence of FITC-labeled conidia on the cuticle of the fruit fly D. melanogaster wildtype strain Canton-S.

Verticals bars represent standard errors. The results of the Tukey-Kramer honest significant difference test are indicated by letters $(\mathrm{p}<0.05)$.

Fig. 2. Initial attachment of FITC-labeled conidia from B. bassiana, A. niger, and $F$. oxysporum on the wings of Canton-S flies 
Scale bars indicate $300 \mu \mathrm{m}$.

Fig. 3. Attachment and persistence of FITC-labeled conidia on the cuticle of the fruit fly D. melanogaster

Black square shapes indicate Canton-S flies treated with $B$. bassiana, white triangles indicate poxn 70 flies treated with $B$. bassiana, and white circle indicate orcol flies treated with $B$. bassiana. Removal efficiency is assessed using the removal index (***: $p$ $<0.01,{ }^{* *}: \mathrm{p}<0.05, *: \mathrm{p}<0.1$, Dunnett's test). Verticals bars represent standard errors.

Fig. 4. Grooming behavior of D. melanogaster strain Canton-S and orco1 induced by olfactory chemicals from the fungus $B$. bassiana. Behavior increase/decrease following fungal odor exposure. The grooming behavior was estimated using the time devoted to grooming during a 3 -minute observation period. $\mathrm{n}=40$ (20 female and 20 male flies). $(* * *: p<0.01, * *: p<0.05, *: p<0.1$, Kruskal-Wallis test).

Fig. 5. Fungal avoidance by the fruit fly D. melanogaster wild type strain Canton-S. $(* * *: \mathrm{p}<0.01, * *: \mathrm{p}<0.05, *: \mathrm{p}<0.1$, Dunnett's test). If PI is low (left), that indicates avoidance and if high (right), that indicates attraction. Horizontal bars represent standard errors.

594

Supplementary Fig. S1. Visitation test model arena.

About 40 flies were introduce to the polystyrene container from the hole at top. The taste preference index (PI) was calculated as (number flies on test substance side - number flies on water side)/(total number of flies). Data were obtained from 10 replicates for each substance.

600

601

602 Supplementary Fig. S2. Attachment and persistence of FITC-labeled conidia from $B$.

603 bassiana on wings of Canton-S, poxn 70, and orco1 flies

604 The conidia removal efficiency was described by the removal index. Verticals bars

605 represent standard errors. The results of Dunnett's tests are indicated by asterisks $(*: \mathrm{p}$ $606<0.05, * *: \mathrm{p}<0.01) . \mathrm{n}=20$ from each sex.

607

608 Supplementary Fig. S3. Design for the rearing of conidia-treated flies used in the 609 bioassays 
610 (a) Assay kits before use. (b) Assays using conidia-treated flies. 
Whole body

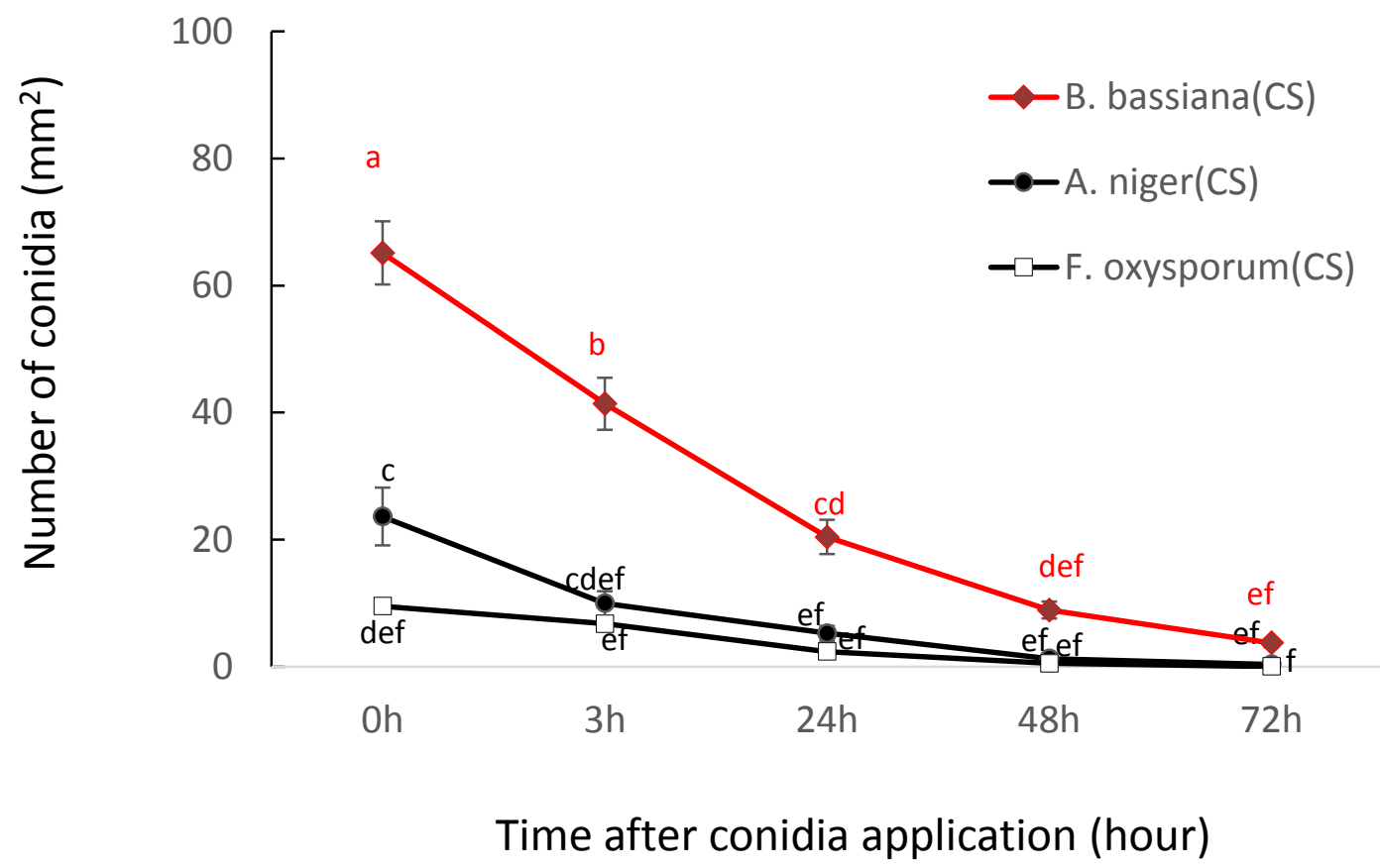

Fig. 1. Attachment and persistence of FITC-labeled conidia on the cuticle of the fruit fly $D$. melanogaster wildtype strain Canton-S.

Verticals bars represent standard errors. The results of the Tukey-Kramer honest significant difference test are indicated by letters $(p<0.05)$ 
Fig. 2. Initial attachment of FITC-labeled conidia from B. bassiana, A. niger, and F. oxysporum on the wings of Canton-S flies

Scale bars indicate $300 \mu \mathrm{m}$.

Fig. 2 
whole body

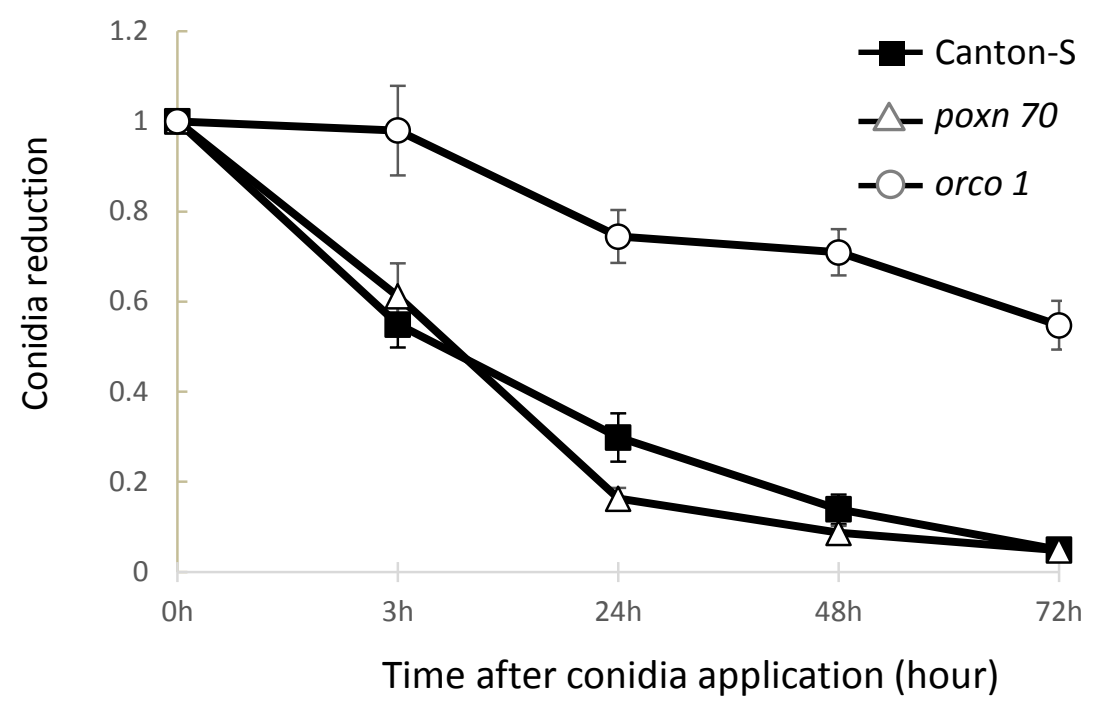

Fig. 3. Attachment and persistence of FITC-labeled conidia on the cuticle of the fruit fly $D$. melanogaster

Black square shapes indicate Canton-S flies treated with $B$. bassiana, white triangles indicate poxn 70 flies treated with $B$. bassiana, and white circle indicate orco1 flies treated with $B$. bassiana. Removal efficiency is assessed using the removal index $\left({ }^{* *}: p<0.01,{ }^{* *}: p<0.05\right.$, $*: p<0.1$, Dunnett's test). Verticals bars represent standard errors. 


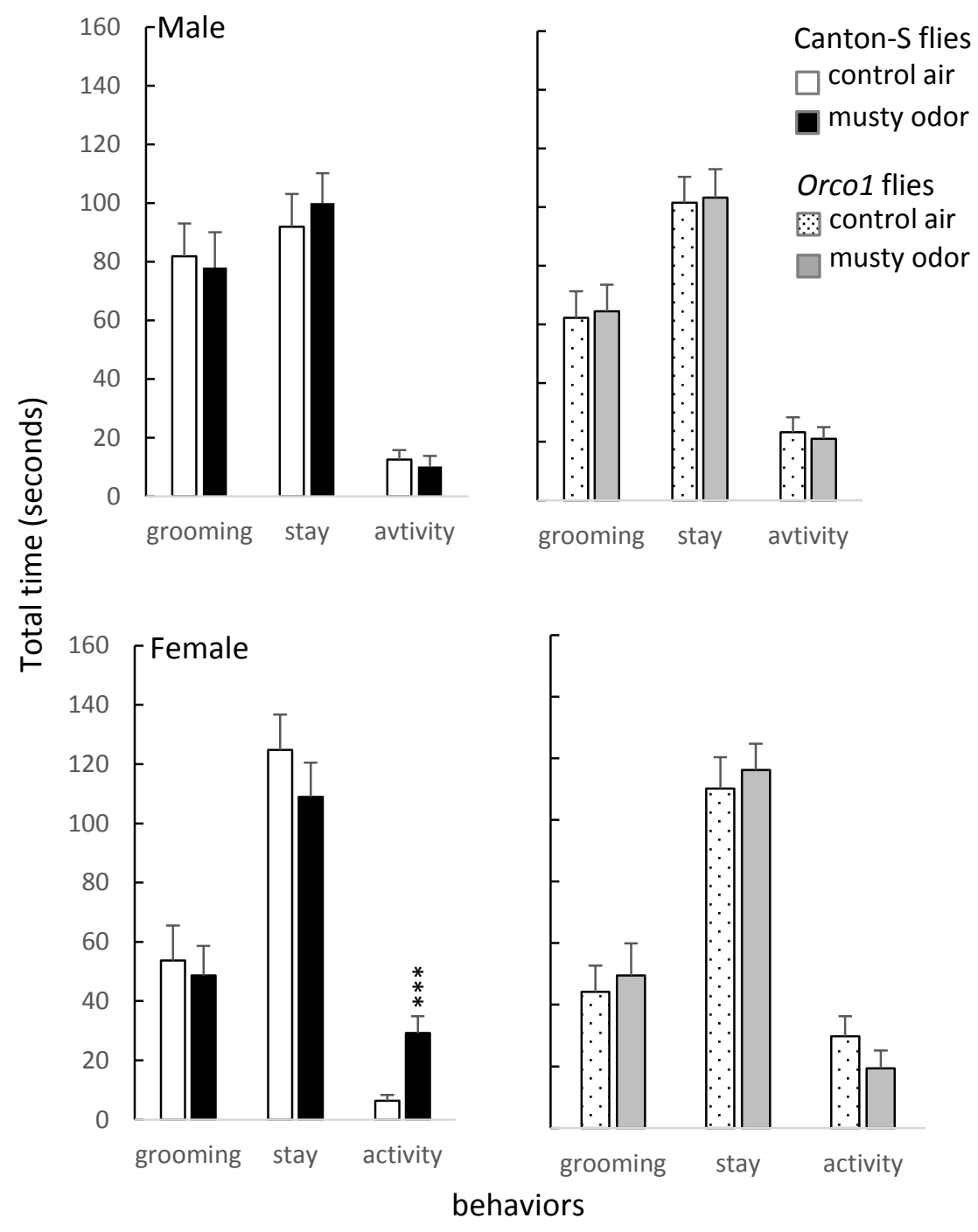

Fig. 4

Fig. 4. Grooming behavior of D. melanogaster strain Canton-S and orco1 induced by olfactory chemicals from the fungus $B$. bassiana. Behavior increase/decrease following fungal odor exposure. The grooming behavior was estimated using the time devoted to grooming during a 3minute observation period. $\mathrm{n}=40$ (20 female and 20 male flies). ${ }^{* * *}: \mathrm{p}<0.01,{ }^{* *}: \mathrm{p}<0.05, *: p$ $<0.1$, Kruskal-Wallis test). 


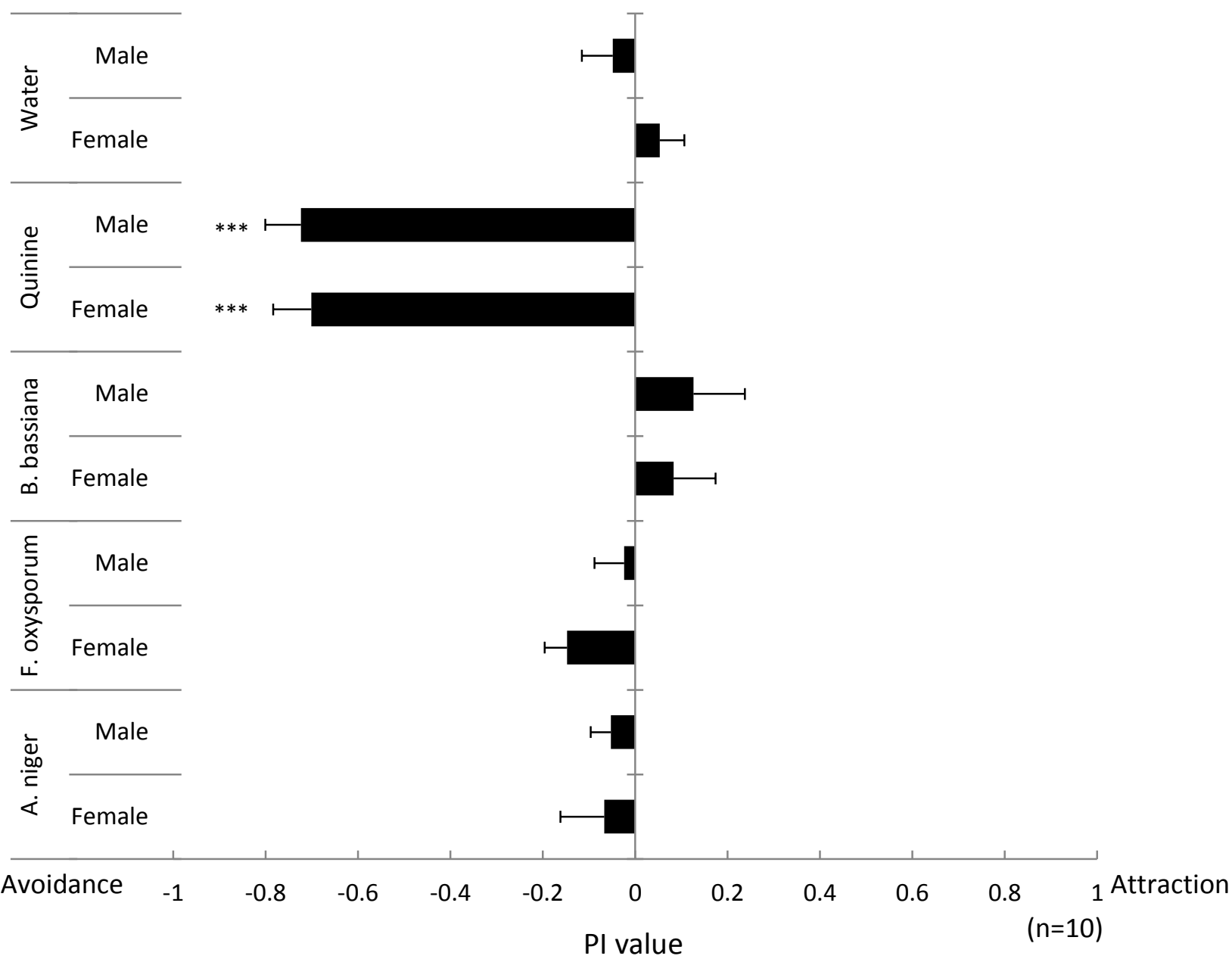

Fig. 5. Fungal avoidance by the fruit fly $D$. melanogaster wild type strain Canton-S.

Visiting preference/aversive responses were examined using the preference index (PI) $(* * *: p$ $<0.01, * *: \mathrm{p}<0.05, *: \mathrm{p}<0.1$, Dunnett's test). If $\mathrm{PI}$ is low (left), that indicates avoidance and if high (right), that indicates attraction. Horizontal bars represent standard errors. 
Supplemental table 1 LD50 of $D$. melanogaster to each fungal strain after 1 week rearing

\begin{tabular}{|c|c|}
\hline $\begin{array}{l}\text { B. Bassiana sensu stricto } \\
\text { Origin: Bombyx mori (Japan) }\end{array}$ & $\begin{array}{l}\text { Laboratory maintain strain F1286 } \\
\text { Last retrieve with Drosophila melanogaster in } 2016\end{array}$ \\
\hline Female + Male & $\geq 4.163 \times 10^{6}$ \\
\hline Female & $=6.250 \times 10^{6}$ \\
\hline Male & $\geq 2.901 \times 10^{6}$ \\
\hline $\begin{array}{l}\text { A. niger } \\
\text { Origin: NBRC\#105649 (U.S.A) }\end{array}$ & $\begin{array}{l}\text { Laboratory maintain strain } 5131 \\
\text { Since } 1990\end{array}$ \\
\hline Female + Male & $>1.633 \times 10^{7}$ \\
\hline Female & $>1.633 \times 10^{7}$ \\
\hline Male & $>1.633 \times 10^{7}$ \\
\hline $\begin{array}{l}\text { F. oxysporum } \\
\text { Origin: Palmier datier (France) }\end{array}$ & $\begin{array}{l}\text { Laboratory maintained strain } 544 \mathrm{H} \\
\text { Since } 1988\end{array}$ \\
\hline Female + Male & $>1.295 \times 10^{7}$ \\
\hline Female & $>1.295 \times 10^{7}$ \\
\hline Male & $>1.295 \times 10^{7}$ \\
\hline
\end{tabular}




\section{Entrance of flies}

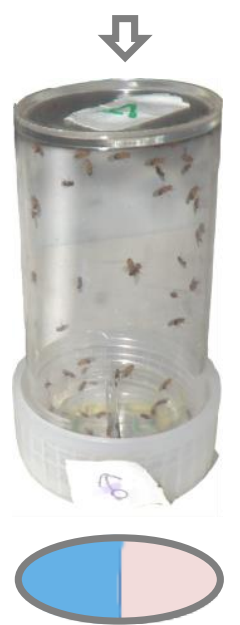

1:control solution 2 : test solution

\section{Fig. S1}

Supplementary Fig. S1. Visitation test model arena.

About 40 flies were introduce to the polystyrene container from the hole at top. The taste preference index (PI) was calculated as (number flies on test substance side - number flies on water side)/(total number of flies). Data were obtained from 10 replicates for each substance. 


\section{Wing}

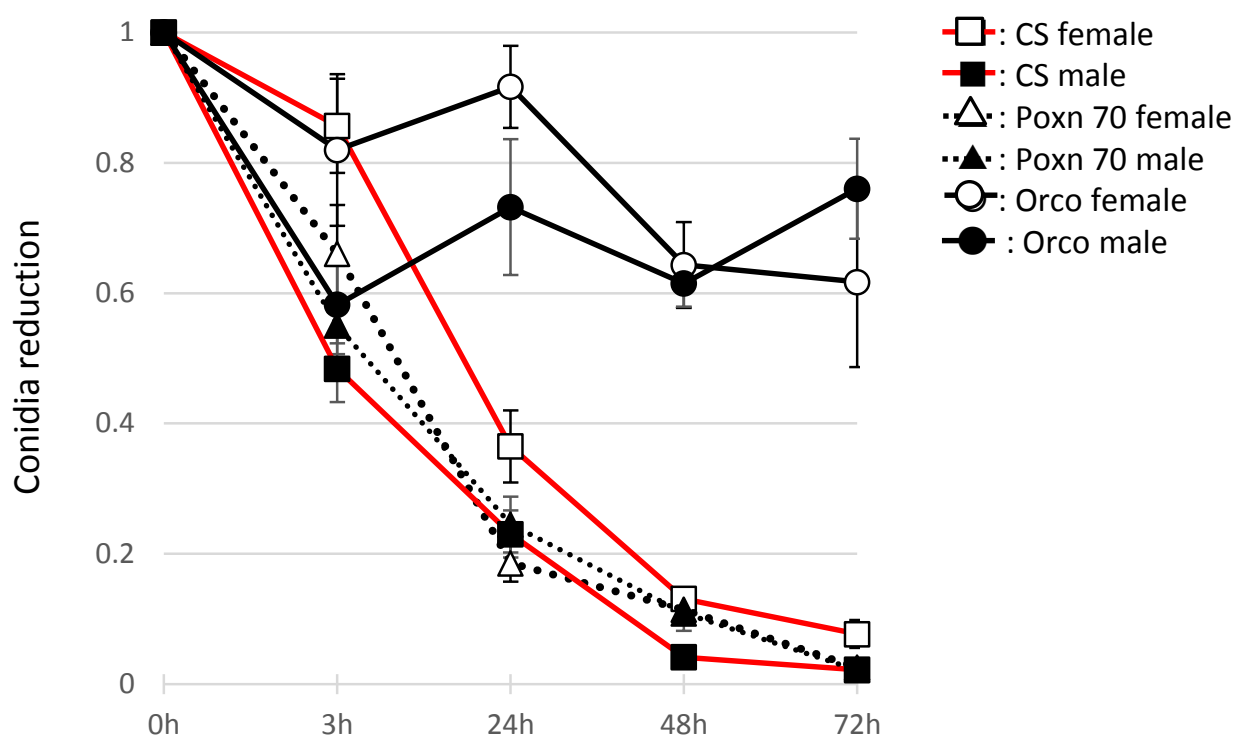

Time after conidia application (hour)

Supplementary Fig. S2. Attachment and persistence of FITC-labeled conidia from B. bassiana on wings of Canton-S, poxn70, and orco1 flies

The conidia removal efficiency was described by the removal index. Verticals bars represent standard errors. The results of Dunnett's tests are indicated by asterisks $\left(*: p<0.05,{ }^{* *}: p<0.01\right)$. $\mathrm{N}=20$ from each sex. 

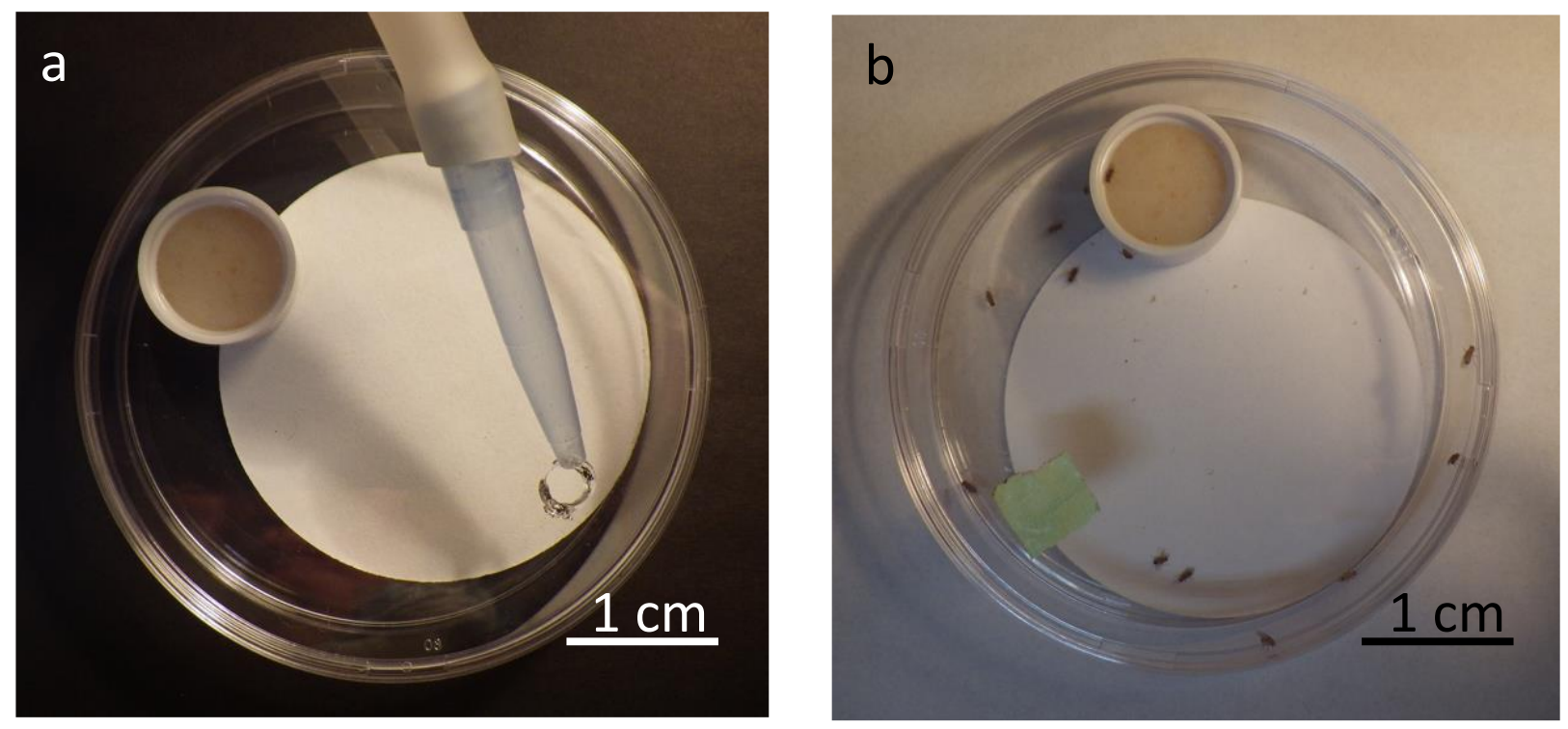

Fig. S3. Supplementary Fig. S3. Design for the rearing of conidia-treated flies used in the bioassays

Assay kits before use. (b) Assays using conidia-treated flies. 